\title{
Erratum to: Rapid Discrimination of F1 Hybrid Seeds from Their Parental Lines and Selection of Protein-Rich Corn Lines for Silage Corn Breeding Using FT-IR Spectroscopy Combined by Multivariate Analysis
}

Seok Hyeon Nahm², Seung Gyun Yang², Suk Weon $\mathrm{Kim}^{3}$, Byung Whan Min"

Erratum to: J. Crop Sci. Biotech. 18(3): 161-169

DOI No. 10.1007/s12892-015-0108-7

In page 1, the authors "Seok Hyeon Nahm², Seung Gyun Yang², Suk Weon Kim³, Byung Whan Min ${ }^{1 *}$ " should be changed as follows:

"Suel Hye Hur ${ }^{1,3}$, Seok Hyeon Nahm², Seung Gyun Yang'2 Suk Weon Kim³, Byung Whan Min"

We apologize to readers for the mistake.

The online version of the original article can be found at

http://link.springer.com/article/10.1007/s12892-015-0108-7

\section{Suel Hye Hur}

School of Ecological \& Environmental System, Kyungpook National University, Sangju 742-711, Korea

Biological Resources Center, Korea Research Institute of Bioscience and Biotechnology (KRIBB), 125 Gwahakro,

Yuseonggu, Daejeon 305-806, Korea

\section{Seok Hyeon Nahm}

Nongwoo Bio Seed Co., Jungdanri, Yeoju, Korea

\section{Seung Gyun Yang}

Nongwoo Bio Seed Co., Jungdanri, Yeoju, Korea

\section{Suk Weon Kim}

Biological Resources Center, Korea Research Institute of Bioscience and Biotechnology (KRIBB), 125 Gwahakro,

Yuseonggu, Daejeon 305-806, Korea

\section{Byung Whan Min}

School of Ecological \& Environmental System, Kyungpook National University, Sangju 742-711, Korea 